Nuclear Physics B96 (1975) 401-406

(c) North-Holland Publishing Company

\title{
A SEARCH FOR PRECURSORS TO EXTENSIVE AIR SHOWERS *
}

\author{
W.E. HAZEN ** \\ Randall Laboratory of Physics, \\ The University of Michigan, Ann Arbor, Michigan 48104 \\ B.R. GREEN, A.L. HODSON and J.R. KASS \\ Department of Physics, \\ University of Leeds, Leeds, England
}

Received 26 May 1975

(Revised 24 June 1975)

\begin{abstract}
No evidence for precursors to cosmic ray air showers was found ("flux" $\leqslant 10^{-8} \mathrm{~cm}^{-2}$ $\mathrm{sr}^{-1} \mathrm{~s}^{-1}$ ) in a sample 2.8 times that of Clay and Crouch and a system noise level several times lower. A simple model leads to a cross section for production $\lesssim 60$ times the total proton-nucleus cross section.
\end{abstract}

A letter by Clay and Crouch [1] reported evidence for a statistically significant bunching of scintillator pulses within the $100 \mu \mathrm{sec}$ interval preceding the arrival of the ordinary component particles of air showers due to primaries of minimum energy $2 \times 10^{15} \mathrm{eV}$. They interpreted their results as possible evidence for the production of tachyons in the interactions of the shower particles or the primary particles with the atmosphere.

We have done a very similar experiment with our air-shower array at Leeds. The portion of the array pertinent to the present experiment is shown in plan in fig. 1. The photomultipliers are EMI 9618. The material above the scintillators averages $2 \mathrm{gm} / \mathrm{cm}^{2}$ of wood and plastic. Scintillators $8 \mathrm{~B}$ and $11 \mathrm{~B}$ are $91 \mathrm{~cm} \times 91 \mathrm{~cm} \times 19 \mathrm{~mm}$, and the scintillators forming the square are $1.2 \mathrm{~m} \times 1.2 \mathrm{~m} \times 38 \mathrm{~mm}$. We triggered transient recording equipment (Data Lab 905) when $2 \mathrm{~S}, 8 \mathrm{~B}$ and $11 \mathrm{~B}$ each registered $\sim 4$ particles $/ \mathrm{m}^{2}$. This trigger condition was designed to be about the same as that of Clay and Crouch but, since our rate was about $10 \mathrm{~h}^{-1}$ (compared to $7 \mathrm{~h}^{-1}$ ) and our $30 \mathrm{~m}$ triangle covers a little less area than their $30 \mathrm{~m}$ square, we would predict that our effective minimum shower energy was somewhat less than theirs. For a

* Supported by a U.S. Atomic Energy Commission Grant to The University of Michigan and by the University of Leeds Physics Department.

** Visiting Professor at the University of Leeds during a portion of the experiment. 


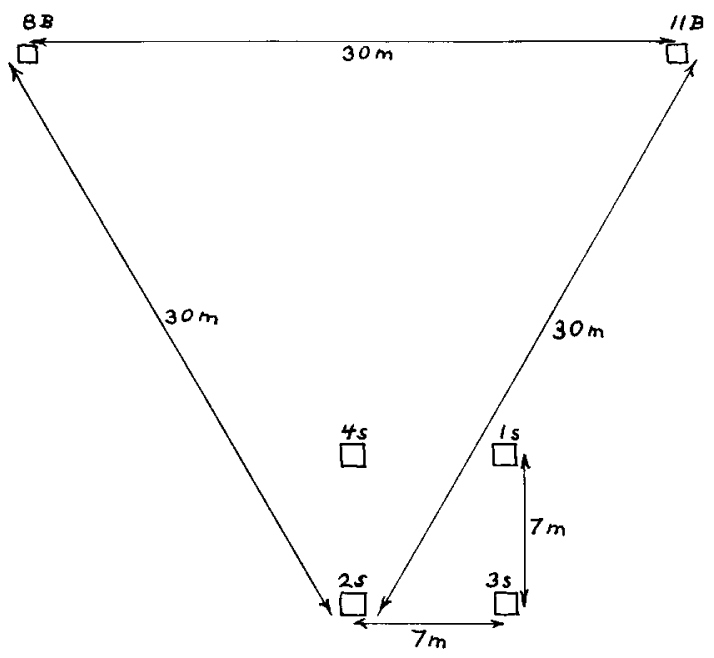

Fig. 1. Plan of scintillator array.

sample of 30 showers in which the particle numbers were recorded and a shower fit made, an effective minimum size of $\sim 10^{5}$ particles was found. About half of the time of this run we recorded pulses from $2 \mathrm{~S}$ and $3 \mathrm{~S}$; the remainder of the time, from $2 \mathrm{~S}$ only. The net running time in Run I was 104 h for 1039 showers. For Run II, scintillator $2 \mathrm{~S}$ was placed immediately above $3 \mathrm{~S}$ and the photomultiplier voltages were raised from $950 \mathrm{~V}$ to $1150 \mathrm{~V}$. Before the increase in voltage, amplifier noise was comparable with scintillator-photomultiplier noise; after the increase it was negligible. The overall gain was increased with resulting saturation for ordinary cosmic ray pulses but easier scanning for small pulses. The net running time was $197 \mathrm{~h}$ for 2366 showers.

The stored information for the $170 \mu \mathrm{sec}$ preceding the shower was transferred to oscilloscope traces and photographed when the shower trigger occurred. After amplification the scintillator pulses had $0.2 \mu \mathrm{sec}$ rise time and $0.5 \mu \mathrm{sec}$ decay time. The amplified pulses were fed to the transient recorder(s) where they were sampled at $0.2 \mu \mathrm{sec}$ intervals with amplitudes digitized into 256 bits per sample. The uncertainty due to amplitude digitizing was negligible. However, the small pulses that we studied were represented by only 4 or 5 time samples and the possibility that no sample lay at the peak resulted in errors in amplitude due to digitizing of ${ }_{-10}^{+0} \%$.

We have first sought evidence for the existence of precursors by following Clay's procedure of scanning the traces (photographs) for the largest pulse(s) and plotting the number distribution for the time of occurrence of the largest pulse(s) appearing within the $160 \mu \mathrm{sec}$ immediately preceding each shower trigger. The choice of $160 \mu \mathrm{sec}$ is based on a model of tachyon production with a high-energy threshold, where, because of the steeply falling energy spectrum of primary cosmic rays, the origin of the tachyons would principally be the first interactions of the primaries. 

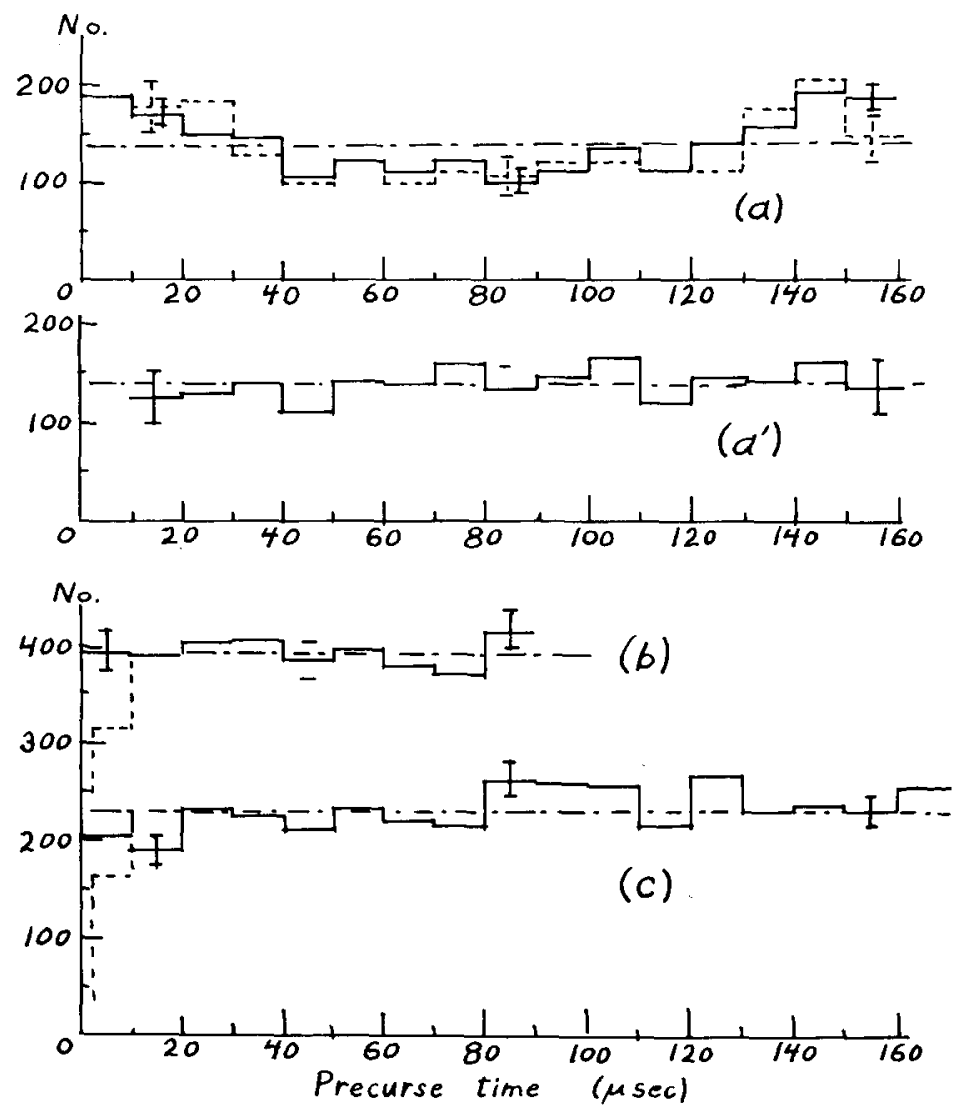

Fig. 2. (a) Frequency of "biggest" pulses in Run I versus time before the arrival of an air shower, solid lines, and time before a two-fold coincidence between single-particle traversals, dotted lines. (The latter is effectively a random choice of trigger time.) The number for the 0 to $10 \mu \mathrm{sec}$ bin was probably accurate, but is omitted because of a priori judgment that there might be ambiguity in the first, 0 to $2 \mu \mathrm{sec}$, sub-bin. The number for the last bin is depleted due to an end effect. The mean for the random trigger was normalized to the mean for the shower trigger. The error bars show representative statistical uncertainties. (a') Run I data replotted using a correction derived from a smooth curve representation of the random trigger distribution. (b) Run II results for the biggest pulse in $100 \mu \mathrm{sec}$. (c) Run II results for the biggest pulse in $170 \mu \mathrm{sec}$.

We would then expect the most probable precursive times to be somewhat less than $100 \mu \mathrm{sec}$.

Only about $3 \%$ of the plotted pulses were significantly above the system noise, which was amplifier noise in Run I, scintillator-photomultiplier noise in Run II. The other $97 \%$ were in the top region of noise $(\gtrsim 0.05$ of the mean muon pulse height 
in Run I and $\gtrsim 0.02$ in Run II) *. Thus, the plotted pulses were almost entirely noise-size pulses, but by inspecting the arrival time distribution for a peak it was possible to ascertain if any significant fraction of noise-size pulses is due to precursors.

Our time-frequency distribution for Run I is shown in fig. 2a. The number of points plotted is about $20 \%$ greater than the number of traces scanned, since we followed Clay in plotting more than one point per trace when there was more than one pulse of "maximum" height. In addition to the large-shower precursor distribution, fig. 2a shows the distribution of precursors of an essentially "random" trigger (dotted). The "random" trigger was actually generated by tiny showers, i.e. a single-particle coincidence between two of our large scintillators separated by $\sim 7 \mathrm{~m}$. This latter distribution (fewer samples) was normalized upward in fig. $2 \mathrm{a}$ by a factor of $138 / 30.9$ for the sake of quantitative comparison, but the error bars shown and the statistical analysis are based on the actual numbers. Statistical analysis shows that the probability that the two distributions are not samples of the same population is only $\sim 0.01$. (For ease of visual judgment, fig. $2 \mathrm{a}^{\prime}$ shows the Run I data corrected by a smooth curve fit to the random trigger calibration data.) Thus, Run I gives no evidence for precursors. The saddle shape of these distributions is due to the fact that the photographed trace had non-negligible $X$-dependence of pulse-height resolution. This resulted from setting the gains low enough in Run I so that most normal cosmic ray pulse maxima could be seen on the oscilloscope.

The time-frequency distribution for Run II is given in fig. 2b. A chi-squared test indicates that the results are satisfactorily represented by a flat distribution ( $40 \%$ probability). Thus we find no evidence for precursors in either run. The combined results include the measured arrival times of $\sim 4000$ "biggest" pulses compared with $\sim 2300$ in the initial observation by Clay and Crouch. Furthermore, our scintillator area is $1.6 \mathrm{~m}^{2}$ compared to $1 \mathrm{~m}^{2}$. Thus, our sample is effectively 2.8 times their sample.

In order to extract some physics from the results, we now consider a model of tachyons as precursors, where we assume that a tachyon somehow produces a pulse only about one order of magnitude lower than ordinary charged particles. An upper limit to the "flux" ** of tachyon precursors is obtained from the scintillator area of $1.6 \mathrm{~m}^{2}$, the effective solid angle of $0.5 \mathrm{sr}$ determined by the shower trigger, the observing time of $190 \mathrm{~h}$ in Run II, and a factor of the order of 100 for the minimum number of precursors required in Run II to make a significant peak in the time frequency distribution. The above factor of 100 is obtained from a model (described below) for the expected arrival times of tachyons and from a conservative criterion for the minimum peak that is statistically significant. Our model for arrival times is that precursive times for tachyons will decrease in likelihood at $\sim 60 \mu \mathrm{sec}$, the tran-

\footnotetext{
* This is about a factor two lower than the noise in Clay's experiment.

** "Flux" as calculated here is simply a convenient way of directly summarizing the experimental observation.
} 
sit time of shower particles from the first interaction layer of the atmosphere. Because of (a) the rapid fall-off of intensity of primary cosmic rays with energy and (b) the large average inelasticity of $\frac{1}{2}$, the probability of creation of particles falls off rapidly with depth in the atmosphere. If we assume that tachyons do not interact strongly, the source of sea-level tachyons will therefore be high in the atmosphere. Consequently, we might expect them to arrive within a time band of wid th of the order $30 \mu \mathrm{sec}$ lying within the first 20 to $70 \mu \mathrm{sec}$ of our observation time. Since the bin width in our frequency distributions is $10 \mu \mathrm{sec}$, the simplest distribution of 100 that would simulate a tachyon peak is $\sim 30$ in each of 3 contiguous bins lying within the 20 to $70 \mu \mathrm{sec}$ interval. This ordering requirement of contiguous results in a factor of $\sim 0.01$ in the probability. The additional factor (determined via a chi-squared simulation) that any 3 bins each have an excess of 30 events is $\sim 0.1$. Thus, our model peak has a probability of $\sim 10^{-3}$ for being generated by fluctutations from a uniform distribution. This is a reasonable criterion for significance of a peak in an exploratory experiment. The resulting upper limit for tachyon "flux" is $\sim 10^{-8} \mathrm{~cm}^{-2}$ $\mathrm{sec}^{-1} \mathrm{sr}^{-1}$.

Our minimum shower size of $\sim 10^{5}$ particles transforms to a primary energy of $1.5 \times 10^{15} \mathrm{eV}$ [2], which is slightly less than the $2 \times 10^{15} \mathrm{eV}$ of Clay and Crouch. However, it seems unlikely that their positive result is due to a rapid rise in probability of precursors between these two energies.

The cross section limit can be estimated by following the method for obtaining quark production cross sections from cosmic ray searches [3]. The same idea is applicable to tachyons, if we assume that they all reach sea level. That is, the path of any tachyon received within the area-solid-angle acceptance of the detector projects back, on the average, to the line of arrival of the primary cosmic ray that produced it. Thus the equivalent source beam is the same as the beam of primaries within the same area-solid-angle acceptance region as the detector but located at the top of the atmosphere. The result is that

$$
\sigma_{\mathrm{t}}=\sigma_{\mathrm{T}} n / 2 I_{0} A \Omega t
$$

where $\sigma_{\mathrm{t}}$ is the tachyon production cross section, $\sigma_{\mathrm{T}}$ the total cross section, $n$ the number of observed tachyons, $A \Omega$ the acceptance of the detector, $t$ the observing time, $I_{0}$ the primary cosmic ray flux, and 2 is Adair's estimate of the number of collisions per primary that are effective in producing quarks, which is a reasonable estimate for tachyons as well. With our effective lower limit for shower size of $N \simeq 10^{5}$ (primary energy $E_{0} \simeq 1.5 \times 10^{6} \mathrm{GeV}$ ) [2],

$$
I_{0}=1.6 \times 10^{-10} \mathrm{~cm}^{-2} \mathrm{sec}^{-1} \mathrm{sr}^{-1} \text {. }
$$

For our tachyon scintillator $A=1.4 \times 10^{4} \mathrm{~cm}^{2}$ and for our shower trigger $\Omega \simeq 0.5$. The running time in Run II was $7 \times 10^{5} \mathrm{sec}$. Since we would have required of the or der of 100 tachyons in order to detect them at all by this method, the lower limit to the cross section that we can set is 


$$
\sigma_{t} \leqslant \sigma_{\mathrm{T}} 100 /\left(2\left(1.6 \times 10^{-10}\right)\left(1.4 \times 10^{4}\right) 0.5\left(7 \times 10^{5}\right)\right),
$$

giving $\sigma_{\mathrm{t}} \lesssim 60 \sigma_{\mathrm{T}}$. We conclude that this is not a very sensitive method for seeking evidence of precursors.

We wish to thank Dr. P.G. Laye and Dr. A. Hughes for the use of their transient recorders and to thank our scanners, Joan Barker, Betty Duncan, Muriel Hunt, Rosalind Jowett, and Jackie Wilson for enthusiastic help.

\section{References}

[1] R.W. Clay and P.C. Crouch, Nature 248 (1974) 28.

[2] K. Greisen, Ann. Rev. Nucl. Sci., vol. 10 (Annual Reviews, Palo Alto, 1960) 63.

[3] F. Ashton, Proc. Int. Conf. on cosmic rays, London 2 (1965) 1108;

R.K. Adair, Proc. 16th Int. Conf. in high-energy physics, 1972, FNAL, Batavia, Ill. 